\title{
Iatrogenic huge intracardiac air embolism during chest computed tomography
}

Seunghoon $\mathrm{Cho}^{1}$, Hancheol Lee ${ }^{2}$, Sanghoon $\mathrm{Shin}^{2}$, Jong-Kwan Park², and Se-Jung Yoon ${ }^{2}$

${ }^{1}$ Division of Internal Medicine, Yonsei University Medical Center, Seoul; ${ }^{2}$ Division of Cardiology, National Health Insurance Service Ilsan Hospital, Goyang, Korea
Received: September 5, 2017

Revised : October 29, 2017

Accepted: October 29, 2017

\section{Correspondence to}

Se-Jung Yoon, M.D.

Tel: +82-31-900-0630

Fax: +82-31-900-0933

E-mail: drpuooh@hanmail.net
A 57-year-old man with hypertension presented to our emergency center for chest discomfort. He had undergone chest computed tomography (CT) in another hospital 4 hours prior for evaluation of a solitary pulmonary nodule. Initial vital signs were $154 / 84 \mathrm{mmHg}$ and 62 beats/min. Electrocardiography, chest radiography, and laboratory test results including arterial blood gas analysis were within the normal ranges. However, large volumes of air in the right atrium, right ventricle, and both main pulmonary arteries were noted
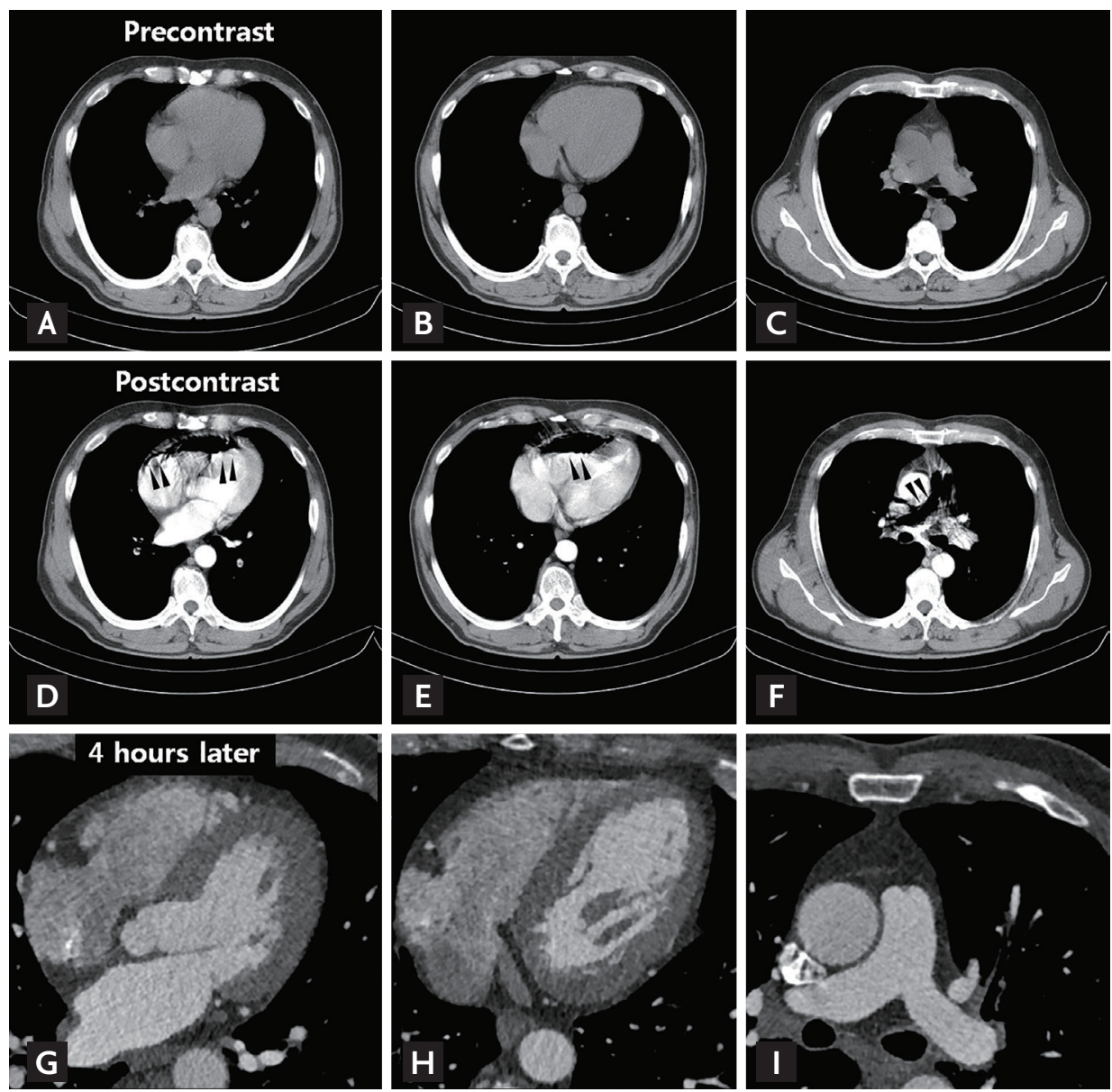

Figure 1. Chest computed tomography showing serial change in an air embolism: (A, B, C) pre-contrast, (D, E, F) post-postcontrast, (G, H, I) 4 hours later. Large amounts of air lodged in the right ventricle, right atrium, and both main pulmonary arteries (black arrowheads). 
in post-contrast phase of chest CT performed in the previous hospital (Fig. $1 \mathrm{~A}-1 \mathrm{~F}$, black arrowheads). The patient complained of only mild chest discomfort but no dyspnea. Cardiac CT was followed for evaluation of the residual air and it revealed no evidence of air in the heart or pulmonary circulation 4 hours later (Fig. 1G-1I). Conclusively, intracardiac air embolism was completely resolved, and the patient spontaneously recovered without any complication.

Accidental venous air embolism can occur during a variety of surgical or diagnostic procedures and usually does not cause any hemodynamic complication. How- ever, introduction of a large amount of air can result in a life threatening situation due to "air lock" in the right ventricular outflow tract or pulmonary artery, leading to collapse of the left ventricle. Early diagnosis and appropriate treatment of this condition can avoid mortality.

Written informed consent was obtained from the patient who participated in this study.

\section{Conflict of interest}

No potential conflict of interest relevant to this article was reported. 